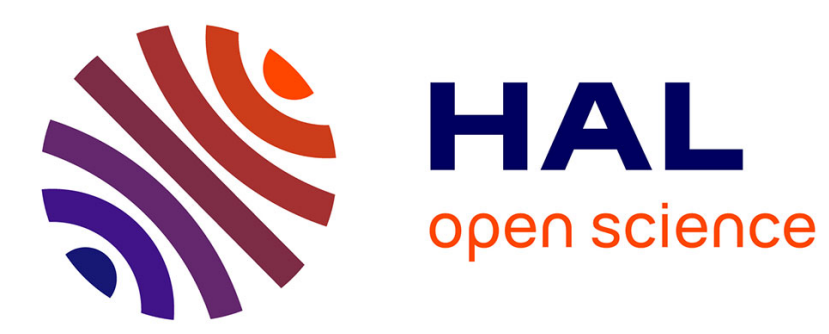

\title{
Traitement d'informations issues de chambres à magnétostriction
}

R. Bruère-Dawson, J.C. Dumont, Ph. Marqueste, M. Rabany, J.P. Turlot

\section{To cite this version:}

R. Bruère-Dawson, J.C. Dumont, Ph. Marqueste, M. Rabany, J.P. Turlot. Traitement d'informations issues de chambres à magnétostriction. Revue de Physique Appliquée, 1969, 4 (2), pp.155-156. 10.1051/rphysap:0196900402015500 . jpa-00243192

\section{HAL Id: jpa-00243192 https://hal.science/jpa-00243192}

Submitted on 1 Jan 1969

HAL is a multi-disciplinary open access archive for the deposit and dissemination of scientific research documents, whether they are published or not. The documents may come from teaching and research institutions in France or abroad, or from public or private research centers.
L'archive ouverte pluridisciplinaire HAL, est destinée au dépôt et à la diffusion de documents scientifiques de niveau recherche, publiés ou non, émanant des établissements d'enseignement et de recherche français ou étrangers, des laboratoires publics ou privés. 


\title{
TRAITEMENT D'INFORMATIONS ISSUES DE GHAMBRES A MAGNÉTOSTRIGTION
}

\author{
R. BRUÈRE-DAWSON, J. C. DUMONT, Ph. MARQUESTE, \\ M. RABANY et J. P. TURLOT, \\ Laboratoire de Physique Atomique, Collège de France.
}

\begin{abstract}
Résumé. - Principe général et description d'un ensemble électronique destiné au traitement d'informations en provenance de chambres à étincelles à magnétostriction. L'implantation du matériel, les résultats et les projets d'extension sont ensuite développés.
\end{abstract}

Abstract. - Comprehensive view and description of an electronic device used for handling the information from magnetostrictive spark chambers. Then follows the general disposition, the results obtained and future developments.

Le problème posé consiste en la digitalisation d'informations provenant de chambres à étincelles à magnétostriction en vue de leur traitement par un calculateur CII 90-40 en ligne. Les informations se présentent de la manière suivante : sur chaque fil de détection, deux signaux de référence, correspondant à la dimension de la chambre, encadrent un troisième signal ayant pour origine l'étincelle [1].

I. Principe adopté. - Les signaux détectés sont mis en forme $(\mathrm{MF})$. Le premier d'entre eux provoque le déversement d'une horloge dans une échelle binaire. Parallèlement, chaque signal, synchronisé avec les impulsions d'horloge, commande la lecture en vol de l'échelle. Le nombre lu est mis en mémoire pour être écoulé en temps voulu vers le calculateur ( fig. 1).

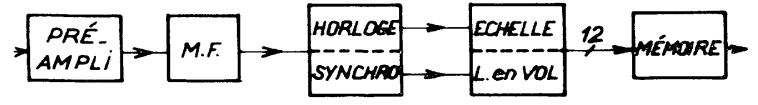

FIG. 1. - Schéma synoptique de l'ensemble électronique ou décodeur.

II. Description détaillée. - Les signaux détectés (qq. 1/10 de $\mathrm{mV}$ ) sont préalablement amplifiés et transmis par câble coaxial d'impédance $100 \Omega$, afin d'obtenir à l'entrée de l'amplificateur un rapport signal sur bruit convenable (gain $=100$, impédance d'entrée $=10 \mathrm{k} \Omega$, impédance de sortie $=100 \Omega$ ). L'amplificateur (linéaire, à gain variable, bande passante maximale $30 \mathrm{MHz}$ ) effectue une première mise en forme. Les signaux amplifiés (largeur $400 \mathrm{~ns}$, temps de montée $200 \mathrm{~ns}$, amplitude $800 \mathrm{mV}$ à $2 \mathrm{~V}$ ) sont ensuite traités de façon à obtenir des impulsions calibrées (16 ns-4 V) tout en conservant la précision de l'information en temps (incertitude $=30 \mathrm{~ns}$ au maximum). L'horloge est pilotée par un quartz oscillant à $20 \mathrm{MHz}$. Le choix de cette fréquence était imposé par la précision désirée. La vitesse de l'ébranlement mécanique dans le fil de magnétostriction étant de $5 \mathrm{~mm} / \mu \mathrm{s}$, il est possible d'obtenir une précision théorique de $0,25 \mathrm{~mm}$ sur la coordonnée. L'échelle est formée de 12 bascules constituant un compteur binaire synchrone à attaque parallèle, de capacité $2^{12}=4096$, ce qui autorise le codage des informations pour une chambre de $1 \mathrm{~m}$.

Un ET programmé permet, lorsqu'un nombre binaire choisi est atteint (correspondant à la plus grande dimension de chambre), de fermer l'accès des impulsions d'horloge du compteur et de remettre à zéro ce dernier. Les impulsions de magnétostriction, mises en forme et synchronisées avec les impulsions d'horloge, se présentent sur des portes commandées par l'état des bascules du compteur à un moment où cet état est bien établi. Suivant cet état, la porte est ouverte ou fermée et la présence ou l'absence d'impulsions en sortie constitue le bit d'information qu'il reste à transmettre au calculateur par l'intermédiaire d'une mémoire tampon. Cette mémoire tampon est constituée par une matrice de tores de ferrite : 12 lignes correspondant aux 12 bits du compteur et 8 colonnes, une par information à mémoriser. L'écriture est possible à une fréquence d'environ $1 \mathrm{MHz}$. La lecture se fait colonne par colonne au rythme d'acquisition du calculateur. Les signaux de lecture sont ensuite mis à niveau logique compatible avec le multiplexeur d'entrée du calculateur.

III. Mise en œuvre sur le terrain et test de l'ensemble. ( fig. 2). - Par une réinjection (INJ) des chambres les unes sur les autres, chaque décodeur traite les informations provenant de deux chambres. L'expérience comprenant 8 chambres, 4 décodeurs ont donc été réalisés. Les lignes à retard utilisées sont du type à magnétostriction (vacoflux $50, \varnothing 2 / 10$ de $\mathrm{mm}$ ) et peuvent atteindre des longueurs de $5 \mathrm{~m}$ ( $1 \mathrm{~ms}$ de retard). Le fil peut être 


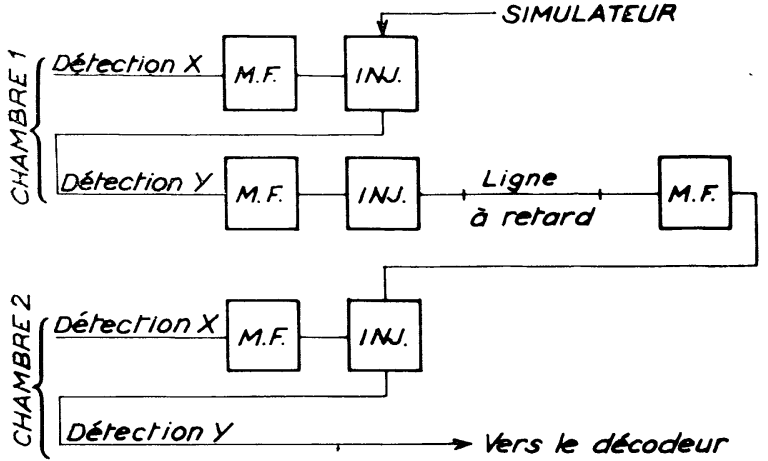

FIG. 2. - Implantation d'un décodeur.

soit tendu sur toute sa longueur, soit coudé, soit enroulé sur un support adéquat. Afin de tester dynamiquement l'appareillage, un dispositif simulant les impulsions de chambres à étincelles (à savoir deux impulsions fixes encadrant un troisième signal distribué au hasard) a été réalisé. Au début du cycle d'accélération, avant l'arrivée des informations véritables, deux tests sont mis en œuvre. Le premier consiste en un déclenchement du simulateur et en l'injection des impulsions obtenues sur chacun des 4 décodeurs. Le second test permet de vérifier la liaison avec le calculateur en affichant à l'entrée du multiplexeur des combinaisons fixes d'in- formations. Le calculateur analyse les données produites par ces deux tests et sort une alerte en cas d'erreurs.

IV. Conclusion. - Cet ensemble électronique fonctionne depuis de nombreux mois auprès du synchrotron à protons « Saturne » de Saclay, dans le cadre d'une expérience de diffusion « $\pi$-protons vers l'arrière ». Cet appareillage a permis l'obtention des résultats présentés dans la communication déjà citée. Cela représente plusieurs semaines de mesures proprement dites. On peut donc considérer que ce fonctionnement a donné satisfaction.

V. Projet de développement. - Les principales limites apportées par cet appareillage sont les suivantes : 1) la plus grande dimension des chambres ne doit pas dépasser $1 \mathrm{~m}$;2) le nombre d'appareils croît avec le nombre des chambres; 3 ) la cadence des informations ne peut dépasser le mégahertz.

Un nouvel ensemble a été étudié et sa réalisation est en cours. Il comporte une mémoire à film mince de 256 mots de 72 bits permettant une écriture toutes les 50 ns. Cette mémoire pourra accepter en parallèle les informations de 29 chambres de grandes dimensions (jusqu'à $4 \mathrm{~m}$ ). L'électronique de synchronisation et de lecture en vol sera adaptée à ces nouvelles conditions et sera totalement réalisée en circuit intégré.

\section{BIBLIOGRAPHIE}

[1] ABILLON (J. M.) et al., ce colloque. 